\title{
Study of tribological properties and lubrication mechanism of surfactant-coated anthracite sheets used as lubricant additives
}

\author{
Hailong LIU ${ }^{1,2}$, Yajing HUANG ${ }^{2}$, Yaozhu WANG ${ }^{2}$, Xiaomin ZHAO $^{2}$, Danqing CHEN $^{2}$, Guohua CHEN $^{2, *}$ \\ ${ }^{1}$ College of Chemical Engineering, Huaqiao University, Xiamen 361000, China \\ ${ }^{2}$ College of Materials Science and Engineering, Huaqiao University, Xiamen 361000, China \\ Received: 29 August 2019 / Revised: 30 October 2019 / Accepted: 18 December 2019 \\ (C) The author(s) 2019.
}

\begin{abstract}
Anthracite sheets were coated by sorbitol fatty acid ester (span80) through ball-milling process. The tribological properties of the span80-coated anthracite sheets as the additive in polyalpha olefin were evaluated through a series of friction tests using a four ball machine. The results revealed that the span80-coated anthracite sheets exhibited excellent dispersion stability in base oil. In addition, compared with base oil, the average coefficient of friction, wear scar diameter, and wear volume of modified oil at a mass fraction of $0.03 \%$ span 80 -coated anthracite sheets decreased by $45.39 \%, 60.13 \%$, and $95.95 \%$, respectively. The oil containing span80-coated anthracite sheets achieved good friction-reducing and anti-wear effects over a wide range of applied loads, temperatures, or rotating speeds. Control experiments were performed as well. The results obtained using span80-coated anthracite sheets were superior to those obtained using pure anthracite. The lubrication mechanism was attributed to the synergistic action of the crystalline and amorphous carbon in anthracite sheets as they formed a protective film and played a mitigative role on the surface of friction pair, which mitigated the wear extent of the friction pair.
\end{abstract}

Keywords: anthracite; tribological properties; lubricant additive; protective film-mending effect

\section{Introduction}

Studies have revealed that approximately $30 \%$ of all primary energy is lost through friction, $50 \%$ of accidents involving mechanical equipment are caused by lubrication failure and/or excessive lubrication, and $60 \%$ of machine parts fail because of wear every year [1]. Lubrication, achieved through the addition of lubricating additives, is one of the most effective approaches for reducing friction and wear. However, conventional lubricant additives contain non-ecofriendly elements, such as sulfur, chlorine, or phosphorus, which cannot reliably meet contemporary production needs. Therefore, developing a green and efficient lubricating additive for the conservation of materials and energy has become an urgent task.

In recent years, considerable research has been conducted on lubricating oil additives, and the results have revealed that nano-elements, such as oxides, sulfides, nitrides, and complexes, nitrogen-containing heterocyclic compounds, and ionic liquids have antiwear (AW), friction-reducing (FR), and anti-corrosion (AC) properties. In addition, carbon-based materials, such as diamond, graphene, fullerene, and carbon nanotubes are recognized as eco-friendly additives. Wang et al. [2] found that graphene can significantly improve the tribological performance of grease under different contact forms. For example, the average friction coefficient was reduced by $15.5 \%$ and the wear loss was reduced by $74 \%$ with the addition of $0.5 \mathrm{wt} \%$ graphene at a load of $200 \mathrm{~N}$. Lee et al. [3] investigated the tribological properties of nano diamonds in oil, and the results revealed that a $0.05 \mathrm{wt} \%$ addition of oleic acid-treated nano diamonds to oil resulted in

* Corresponding author: Guohua CHEN, E-mail: hdcgh@hqu.edu.cn 
excellent friction-reducing and anti-wear properties with the coefficient of friction (COF) reduced by $23 \%$. Table 1 shows the types, roles, and lubrication mechanisms of the reported lubricant additives. Although these carbon-based materials have better anti-wear and anti-friction performances, their disadvantages such as high cost or complex preparation processes limit their development for industrial applications. Thus, it is crucial to find other lubricating oil additives with excellent anti-wear and friction-reducing properties that can overcome the shortcomings of the aforementioned materials.

As a naturally abundant carbon-based material, coal, is primarily used to generate energy. Therefore, comprehensive utilization of coal is particularly important. Anthracite has the advantages of having a high carbon content, high mechanical strength, good thermal stability, and low sulfur content, which make it stand out among many kinds of coal. In previous literature reports, anthracite was utilized to prepare coal-based lithium anode materials [18], coal-based nanomaterials [19, 20], coal-based polymer composites [21], anthracite adsorbents [22] and so on. However, studies on the utilization of anthracite as a lubricant additive, particularly its effect on the tribological behavior of base oil, remain very limited.

As anthracite has a large aromatic lamellar structure, good directionality, and a two-dimensional microcrystalline lamellar structure similar to that of graphite $[23,24]$, it seems reasonable therefore to test anthracite as lubricating oil additive. However, anthracite contains many polar functional groups, such as phenolic hydroxyl, carboxy, and methoxyl groups, which make it susceptible to agglomeration in non-polar oil, thereforem, it is difficult to directly use it as an additive for oil-based lubricants.

Table 1 Summary of types and properties of particles used as lubricant additives.

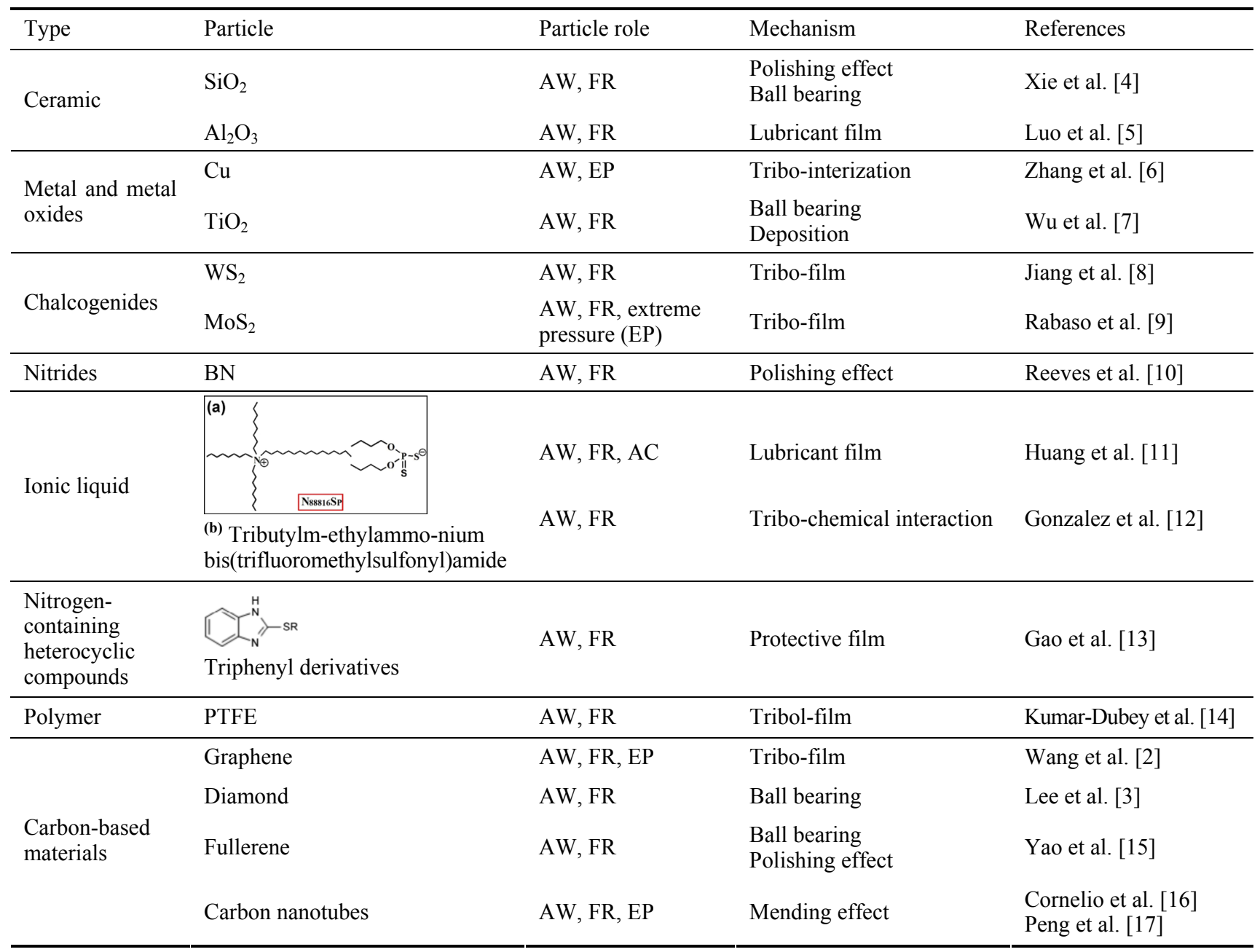


Agglomeration is a major hindrance to the dispersion of additives in a fluidic medium. It has been proven that colloidal stabilization of an additive in a base oil can be achieved by adding a surfactant to the oil [25]. Zhang et al. [26] first washed graphene with petroleum ether and $\mathrm{N}$-methylpyrrolidone to enhance its lipophilicity and dissolved it in 150SN base oil with polyisobutene succinimide as the dispersing agent. Uniform and stable lubricating oils using different mass fractions of graphene $(0.001 \%$ and $0.005 \%)$ were obtained.

These results indicate that selecting an appropriate surfactant is essential to achieve desired frictionreduction and anti-wear properties. Experiments revealed that span 80 can be used to solve the dispersion problem of anthracite in a base oil. The chemical formula of span 80 is $\mathrm{C}_{24} \mathrm{H}_{44} \mathrm{O}_{6}$, and its chemical structure is illustrated in Fig. 1. It can be seen that the span 80 molecule possesses an oleophobic head and a lipophilic chain. In a fluidic medium containing a non-ionic surfactant, anthracite dispersion is stabilized by the steric repulsion force [27]. When anthracite sheets are dispersed in a base oil, the oleophobic head of span 80 is adsorbed onto the anthracite sheets because of van der Waals forces and hydrogen bonds, while the lipophilic chain extends into the oil. Therefore, the anthracite sheets are coated with the surfactant molecule by adhesion. The interaction between the lipophilic chains of the adhered molecules from adjacent anthracite sheets can generate a steric repulsion force, preventing the anthracite sheets from reaggregating with each other.
In this study, a series of tribological tests were conducted to investigate the effect of span80-coated anthracite sheets as an additive on the lubrication properties of a base oil. The lubrication mechanism of anthracite in improving the tribological properties of base oil is also discussed.

\section{Experimental}

\subsection{Materials}

Anthracite and graphite power were provided by Fujian Tianhushan Energy Industry Co., LTD and Fujian Kaili Specialty Graphite Co., respectively. PAO40 base oil was supplied by Beijing Sunright Trade Co., Ltd. The surfactant span 80 was purchased from the Aladdin Industrial Co., Ltd. The physicochemical properties of PAO40 base oil and span 80 are given in Tables 2 and 3, respectively. Cyclohexanone, ethanol, and hydrofluoric acid, all of analytical reagent grade, were purchased from the Sinopharm Chemical Reagent Co., Ltd.

\subsection{Pretreatment of the anthracite}

Commercially available anthracite often contains many impurities, such as inorganic salts and inorganic minerals. If these impurities are not removed, they may not only make anthracite difficult to disperse in the base oil, but also considerably affect its tribological performance. Therefore, in this study, commercially available anthracite powder was mixed with distilled water, and the resulting blend was milled for $3 \mathrm{~h}$

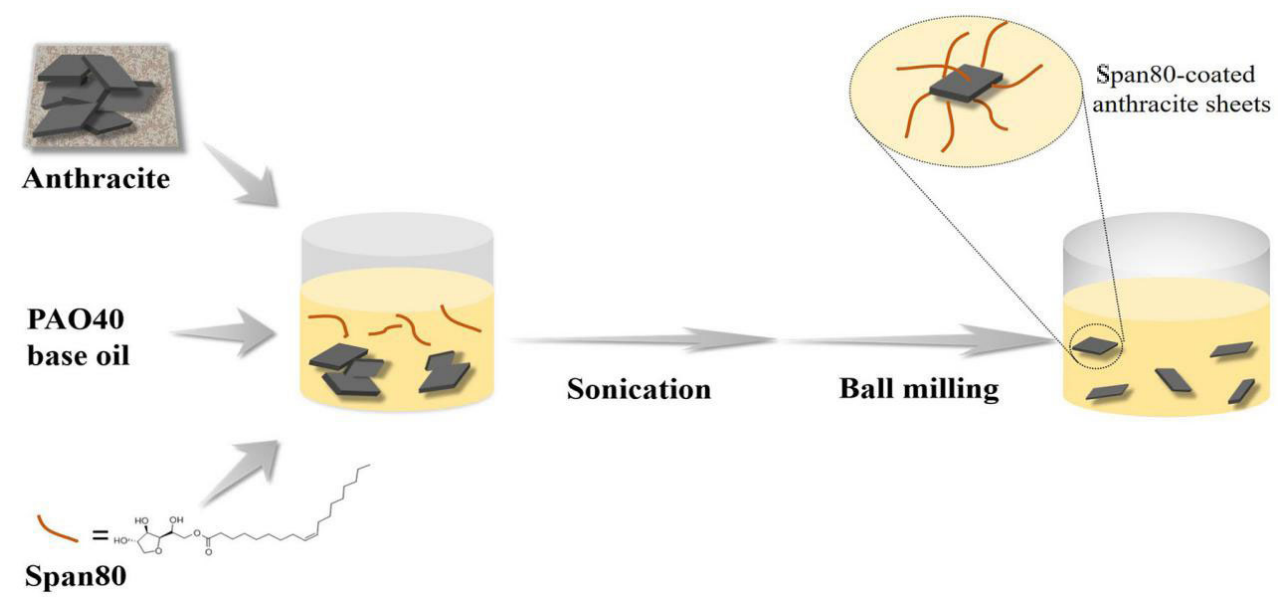

Fig. 1 The schematic process flow for fabrication of oil containing span80-coated anthracite sheets. 
Table 2 Typical characteristics of PAO10 as base oil.

\begin{tabular}{ll}
\hline Item & Value \\
\hline Kinematic viscosity $\left(\mathrm{mm}^{2} / \mathrm{s}\right)$ at $40{ }^{\circ} \mathrm{C}$ & 62.9 \\
Kinematic viscosity $\left(\mathrm{mm}^{2} / \mathrm{s}\right)$ at $100{ }^{\circ} \mathrm{C}$ & $9-11$ \\
Viscosity index & $\geq 128$ \\
Pour point $\left({ }^{\circ} \mathrm{C}\right)$ & $\leq-60$ \\
Flash point $\left({ }^{\circ} \mathrm{C}\right)$ & $\geq 230$ \\
Acid value $(\mathrm{mgKOH} / \mathrm{g})$ & 0.02 \\
Bromine value $(\mathrm{gBr} / 100 \mathrm{~g})$ & 0.01 \\
Monomer & Polydecene \\
Color & Yellowish \\
\hline
\end{tabular}

Table 3 Physical properties of span 80.

\begin{tabular}{ll}
\hline Item & Value \\
\hline Hydroxyl value $(\mathrm{mgKOH} / \mathrm{g})$ & $190-220$ \\
Koettstorfer value $(\mathrm{mgKOH} / \mathrm{g})$ & $140-160$ \\
Acid value $(\mathrm{mgKOH} / \mathrm{g})$ & $\leq 10$ \\
Water content $(\%)$ & $\leq 1.5$ \\
HLB value & $\sim 4.3$ \\
Extrinsic feature & Amber and melicera oil \\
\hline
\end{tabular}

using a planetary ball mill machine. The obtained slurry was extracted using cyclohexanone to remove the inorganic minerals. After the obvious delamination appeared in the static setting, the upper black slurry was collected first, then washed with ethanol three times, and finally stirred in dilute hydrofluoric acid ( $5 \mathrm{wt} \%$ ) for $24 \mathrm{~h}$ to remove the inorganic salts. Finally, the resulting anthracite was filtered and dried in an oven at $80^{\circ} \mathrm{C}$. The morphology and structure of the product were characterized using scanning electron microscopy (SEM), transmission electron microscope (TEM), X-ray powder diffraction (XRD), and Raman spectrometry.

\subsection{Preparation of oil containing span80-coated anthracite sheets}

First, a certain proportion of surfactant and the above product was added to the base oil. The mixture was processed under bath sonication; then, the oil containing span80-coated anthracite sheets was prepared via the ball milling process. The flow scheme for preparation of the oil containing span80-coated anthracite sheets is shown in Fig. 1. For comparison, graphite was also used as an additive, and oil containing span80-coated was obtained by the same method as described above. In addition, the ball milling of the base oil and anthracite was also performed so that a contrastive analysis could be conducted. Finally, the dispersion stability of the additives in oil was recorded under different conditions by a camera.

\subsection{Tribological properties of different oils}

The COF, wear scar diameter (WSD), and wear volume (WV) of the oil containing span80-coated anthracite sheets were examined using a MS-10A four ball machine (Xiamen Tenkey Automation Co., China) and compared with those of the base oil and oil containing anthracite and span80-coated graphite. The friction and wear tests were carried out according to the ASTM D4172-82 standard and were conducted at a rotating speed of 1,200 rpm under a constant load of $150 \mathrm{~N}$ for $1 \mathrm{~h}$; a temperature of $75 \pm 2{ }^{\circ} \mathrm{C}$ was maintained throughout the entire testing process. The balls (diameter of $12.7 \mathrm{~mm}$ ) used in the tests were made of GCr15 bearing steel (AISI 52100) with a hardness of $64 \mathrm{HRC}$. In addition to the aforementioned test conditions, we changed the operating applied load, rotating speed, and temperature to study the tribological performance of anthracite in more detail when other conditions remained unchanged. The specific experimental conditions are presented in Table 4.

The WSDs on the three lower steel balls were measured with an optical microscope, and the ACOFs was determined by the average value of the COFs. Each tribological tests were repeated at least thrice. The morphologies of the worn steel surfaces after the friction tests were observed with SEM, and the WVs were measured with a three-dimensional optical profiler. EDS and Raman spectroscopy were performed to determine the elemental and material composition of the worn steel surfaces.

Table 4 Experimental conditions of tribological tests.

\begin{tabular}{lcccccc}
\hline Item & \multicolumn{6}{c}{ Value } \\
\hline Rotating speed (rpm) & 200 & 800 & 1,200 \\
Temperature $\left({ }^{\circ} \mathrm{C}\right)$ & 27 & 50 & 75 & 100 & & \\
Applied load $(\mathrm{N})$ & 50 & 100 & 150 & 200 & 250 & 300 \\
\hline
\end{tabular}

\section{Results and discussion}

\subsection{Characterization of anthracite sheets}

Figure 2 shows the morphology and internal structure 

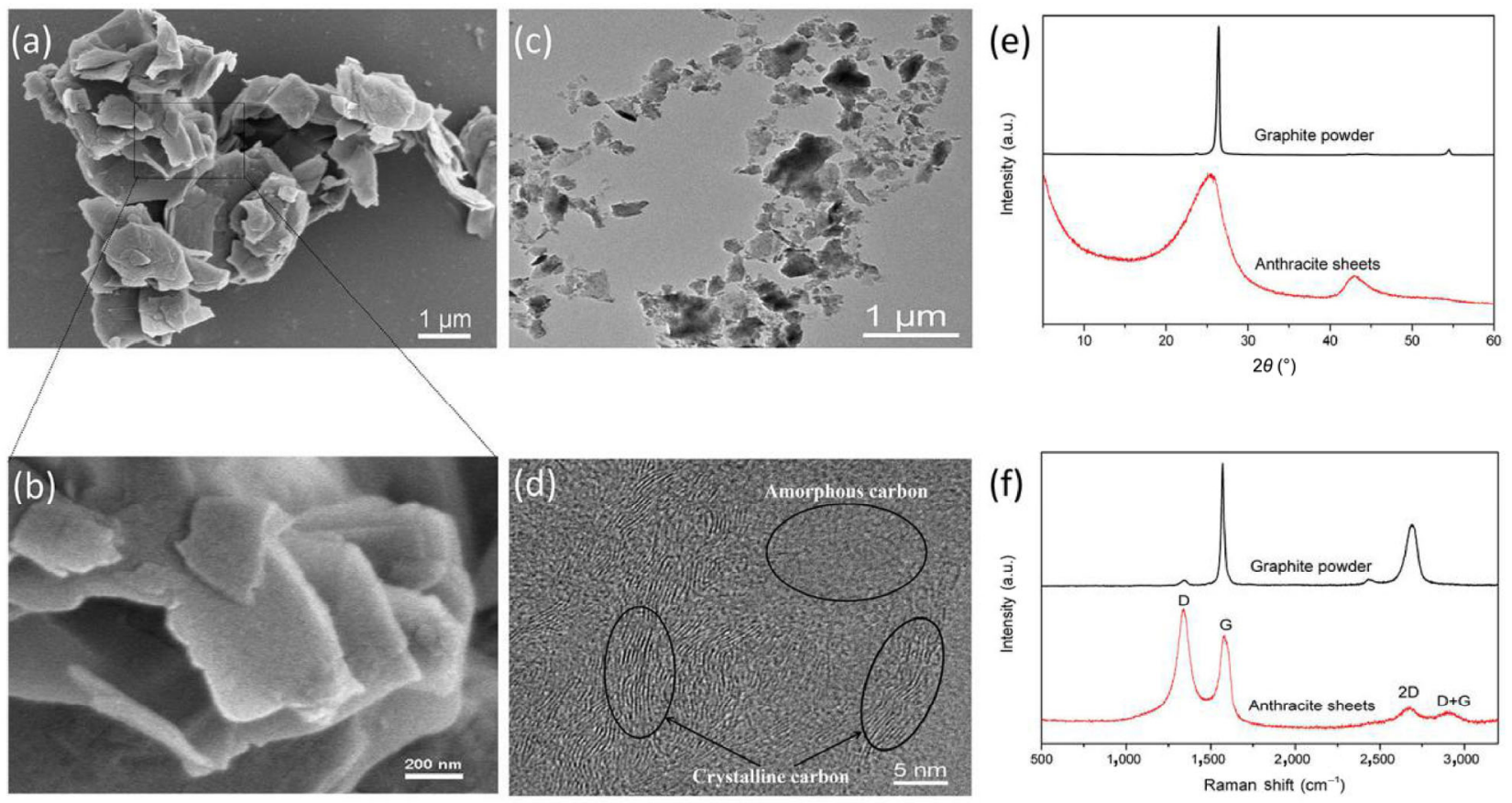

Fig. 2 SEM (a, b) and TEM (c, d) images of the anthracite sheets; X-ray diffraction (e) and Raman spectra (f) of the anthracite sheets and graphite powder, respectively.

of anthracite. The SEM images shown in Figs. 2(a) and 2(b) reveal that the morphology of anthracite possesses a clear laminated structure. A large amount of amorphous carbon and nano-crystalline domains exist in the anthracite, as shown in Fig. 2(d), at the same time. The XRD pattern of the anthracite revealed that it has a higher background strength, which also indicates that the anthracite contains a large amount of amorphous carbon (Fig. 2(e)). Meanwhile, the (002) peak at about $26^{\circ}$ and the (100) peak at about $42^{\circ}$ suggest that the anthracite has a lamellar structure similar to that of graphite. Figure 2(f) is the typical Raman spectra of the anthracite and graphite powder. Generally, the D peak $\left(1,350 \mathrm{~cm}^{-1}\right)$ can be attributed to the disordered graphite lattices, and the $G$ peak $\left(1,580 \mathrm{~cm}^{-1}\right)$ can be attribute to the ideal graphitic lattices. The degree of graphitization disorder $\left(I_{\mathrm{D}} / I_{\mathrm{G}}\right)$ of graphite and anthracite is 0.02 and 1.24, respectively [28]. The higher ratio $\left(I_{\mathrm{D}} / I_{\mathrm{G}}\right)$ indicates that anthracite sheets possess a high degree of defects [29], including structural defects or the presence of certain functional groups at the edge of the anthracite molecule. The $G$ peak indicates that the anthracite sheets have a graphitization degree [30]. These results are consistent with those in the relevant literature reports [31,32].

\subsection{Dispersive stability of oil containing span80- coated anthracite sheets}

The time-dependent dispersion of additives in the base oil was recorded by camera pictures, as shown in Fig. 3(a). It can be seen that the lamination of lubricating oil without span 80 was obvious, and almost all additives had precipitated one day after deposition, while the appearance of the lubricating oil with span80-coated anthracite sheets did not exhibit any change, and there was no deposition at the bottom after 90 days of storage. Meanwhile, as shown in Fig. 3(b), although oil containing span80-coated anthracite sheets was subjected to a long period of high speed centrifugation, it remained black in appearance, and a small quantity of precipitation had settled at the bottom. The above results indicate that span80-coated anthracite exhibited excellent dispersion stability in the base oil; thus, further investigation of its friction performance was performed.

\subsection{Tribological behaviors of oil containing different additive}

Bartz et al. [33] found that the solid additives in liquid lubricants had beneficial effects on the anti-friction 
properties of a lubricant and indicated that there exists an optimal concentration of solid additives. Therefore, series dispersions of different content were prepared to obtain the optimal additive content that would result in optimum tribological behavior. Figures 4(a) and 4(b) show that the ACOF and WSD of oil with different additives can be reduced by varying degrees. When the span80-coated anthracite sheets with a mass concentration of 0.03 were added, the ACOF and WSD reached their lowest values, which were $45.39 \%$ and $60.13 \%$ lower than those of the base oil, respectively.

The COF is a function of friction time, and the results for the different lubricants are shown in Fig. 4(c). Under the same test conditions, the COF curve of the PAO40 base oil maintained an upward trend with time. This is because the oil film provided by the base oil ruptured after a long period of wear, which led to direct contact between the friction pairs and thus an increase in the COF $[34,35]$. The COF curve of the oil
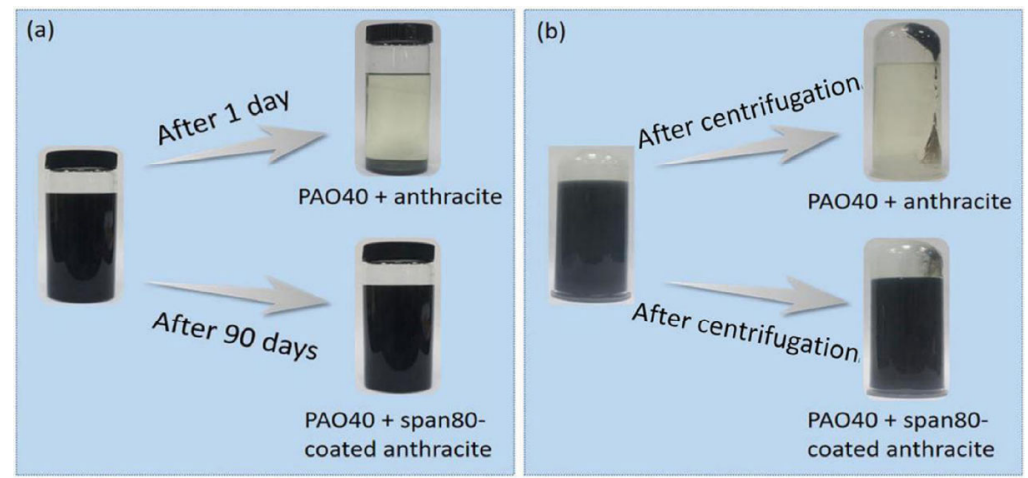

Fig. 3 Camera photographs of oil containing anthracite or span80-coated anthracite sheets: (a) after a storage of 1 day and 90 days, respectively; (b) before and after centrifugation $(10,000 \mathrm{rpm} / \mathrm{min}, 60 \mathrm{~min})$, respectively.
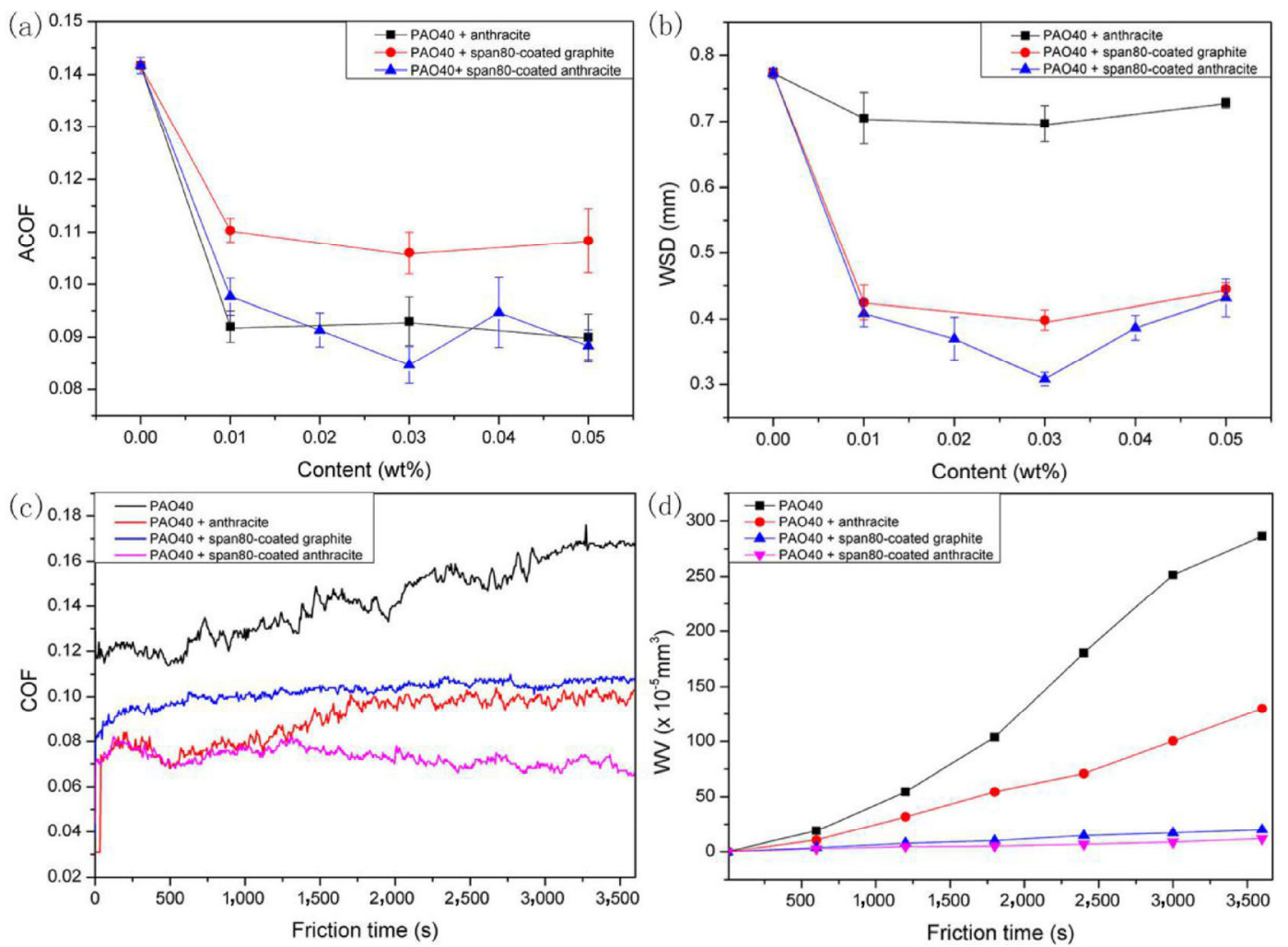

Fig. 4 Effect of additive content on average coefficient of friction (a) and wear scar diameter (b); effect of friction time on coefficient of friction curves (c) and wear volume (d). (four-ball, 1,200 rpm, $150 \mathrm{~N}$, and $1 \mathrm{~h}$ ) 
containing span80-coated graphite could be maintained at about 0.07 , owing to the lubricity of graphite $[36,37]$. The COF curves of oil containing span80-coated anthracite sheets and anthracite sheets were similar, i.e., they could be maintained at a low level at the beginning of the friction experiment. However, with an increase in time, the COF of oil containing anthracite sheets began to increase slowly, finally reaching approximately 0.100 . This is mainly because anthracite could only maintain a short dispersion stability state, and when the oil was stirred at a high speed, anthracite separated from the oil so quickly so that it could not have an anti-wear and anti-friction effect on the friction pair. Figure 5(a) showed camera photos of the oil before and after it was stirred. The appearance of the oil became closer to that of the base oil. Figure 5(b) shows that many additives settle at the bottom of the oil cup. In contrast, span80-coated anthracite sheets could maintain a stable state for a long time in oil, which prevented the occurrence of the above situation. As a result, the COF of oil containing span80-coated anthracite sheets remained basically unchanged. This result illustrates that span80-coated anthracite could enter the friction pair, penetrate the defective parts, repair them, and form protective deposition film thereon. Thus, a smooth COF curve was obtained.

Furthermore, we calculated the WV for the corresponding WSD using the following equations to show the tribological properties of different oils [38]:

$$
\begin{gathered}
V=(\pi h / 6)\left[\left(3 d^{2}\right) / 4+h^{2}\right] \\
h=r-\left(r^{2}-d^{2} / 4\right)^{1 / 2}
\end{gathered}
$$

where $d$ is the WSD, and $r$ is the radius of the steel ball. Figure 4(d) shows the calculated WVs of different lubricating oils as a function of friction time. With an increase in the friction time, the WV of PAO40 base oil increased significantly, but that of oil containing span80-coated anthracite sheets was almost unchanged. Compared with WV of steel ball by base oil lubrication, that of oil containing span80-coated anthracite sheets decreased by $95.95 \%$ (from 286.36 to $11.60 \times 10^{-5} \mathrm{~mm}^{3}$ ), indicating that the anthracite sheets played an important role in reducing wear.

Figure 6 shows that the planar and 3D morphologies of the worn steel surfaces lubricated with base oil alone, oil containing anthracite, and oil containing

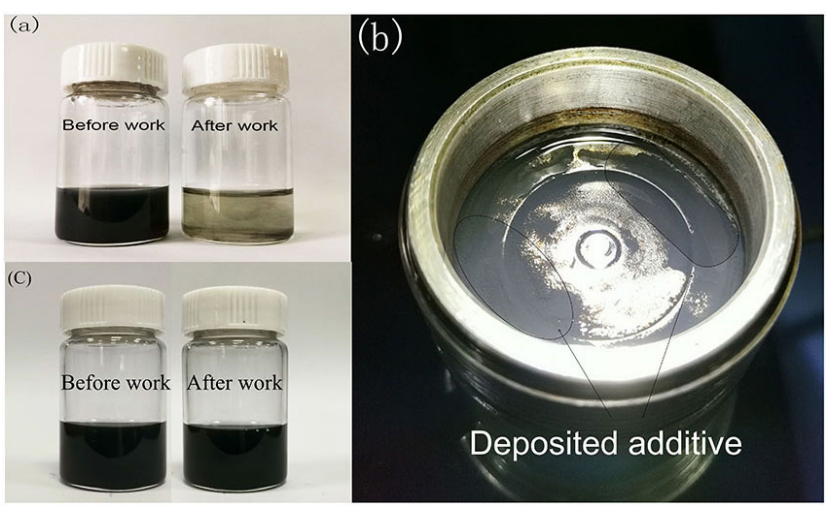

Fig. 5 Camera pictures of: (a) oil containing anthracite before and after work; (b) the bottom of the oil cup after work; (c) oil containing span80-coated anthracite sheets before and after work.

span80-coated anthracite sheets additive. It can be seen that oil containing span80-coated anthracite sheets provides a smaller wear scar, wear depth, and wear volume than base oil alone and oil containing anthracite. This result further verifies that span80coated anthracite sheets can effectively improve the tribological properties of base oil.

Figure 7 shows the tribological properties of oil containing span80-coated anthracite sheets and base oil under varied applied load. Figures 7(a)-7(c) reveal that the fluctuation of the COF curve was significant under lower applied loads. As the applied load increased, the fluctuation reduced, which met the actual production needs. The increase in the applied load resulted in greater serve wear. As shown in Fig. 7(d), for the same kind of additive, the WSD increased with an increase in load. However, for different additives, the percentage of the increase in the WSD was different with an increase in load, which resulted in the oil containing the span 80 -coated anthracite sheets having a reduction of only $38.30 \%$ in the WSD at a lower load and up to $60.22 \%$ at a higher load than that of pure base oil, indicating that span80-coated anthracite could significantly improve the wear resistance ability of base oil at higher applied loads. The main reason for this phenomenon is that, with increasing load, the base oil cannot provide a complete protective film between the friction pairs, resulting in a sharp increase in the WSD, but for the oils containing span80-coated anthracite sheets, it can continue to provide a protective film. For this reason, the WSD was not affected considerably. 

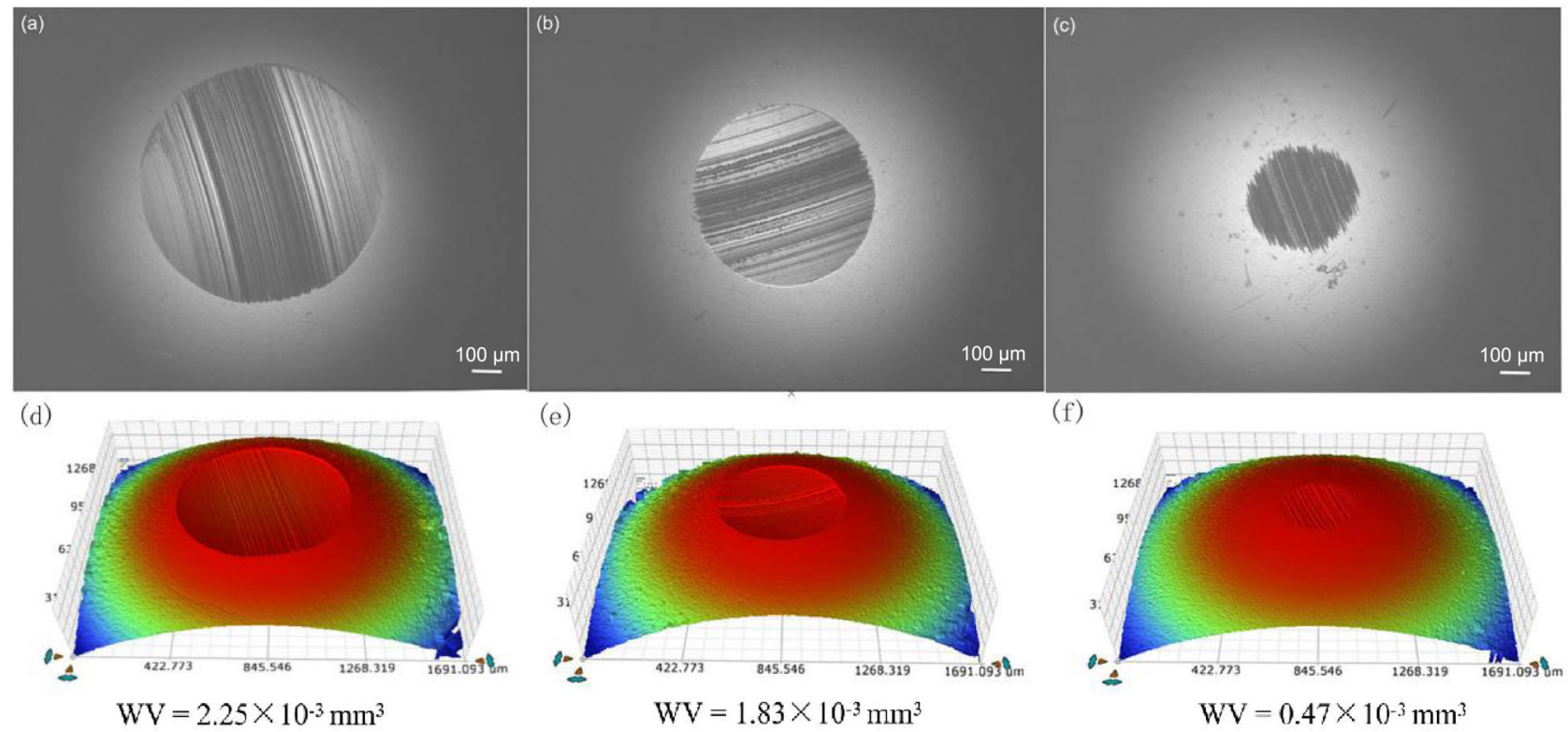

Fig. 6 The planar and 3D morphologies of the worn surface lubricated with: base oil (a, d); oil containing anthracite (b, e); and oil containing span80-coated anthracite (c, f).
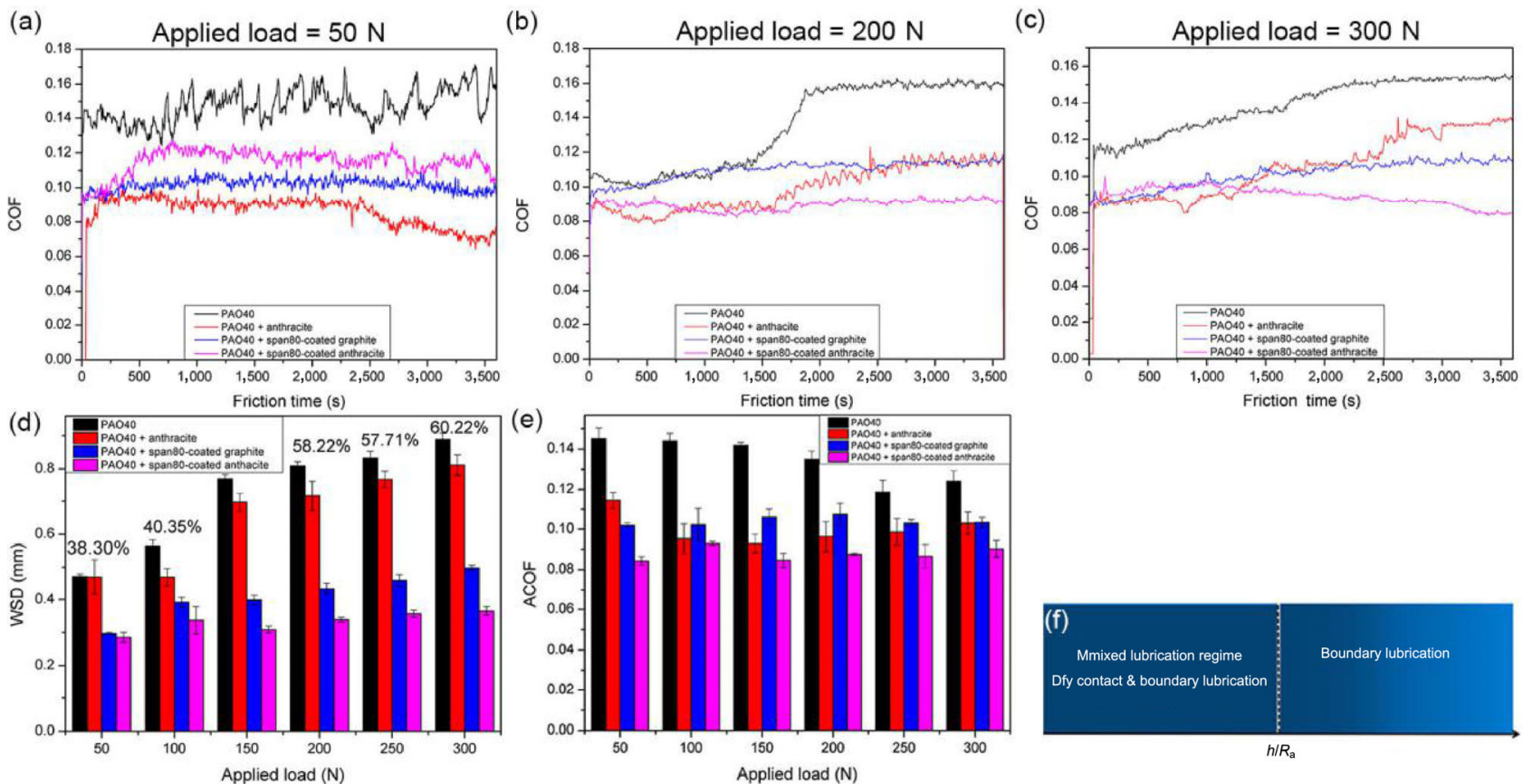

Fig. 7 (a-c) Effect of applied load on COF curve; effect of applied load on: (d) WSD; (e) ACOF; (f) lubrication regime transition.

However, the ACOF exhibited different trends with an increase in applied load. It could be observed that, for the base oil, the ACOF was higher under the lower applied load, and when the applied load increased, the ACOF tended to first decrease and then increase. The phenomena can be explained by the lubrication regime transition [39] shown in Fig. 7(f). As the strength of its oil film was so low that the oil film was prone to rupture, the base oil could not easily form a continuous lubricating medium between the friction pairs; as a result, the ACOFs of the entire interval were not high. Under the lower applied load, because the test process was vulnerable to interference from the external environment (as shown in Fig. 7(a), the fluctuation of the COF was acute), the possibility of direct contact between the microscopic peaks of the 
friction pair surface increased, and the probability of dry friction during mixed lubrication also increased, which increased the ACOF. For oil containing additives, the ACOF remained relatively stable. This is because the protective film formed by the additive could reduce the surface roughness of the friction pair, which led to a boundary lubrication state; therefore, the ACOF remained relatively stable [40].

In practical applications, an additive is expected to enhance the lubricating properties under various operating conditions. Thus, we investigated the effects of different applied loads, rotating speeds, and temperatures on the performance of oil containing span80-coated anthracite sheets under other experimental conditions. The lubricating effects of the oil containing span80-coated anthracite sheets under different temperatures and rotating speeds were determined, as shown in Table 5. The results revealed

Table 5 Tribological properties of oil containing anthracite sheets under different conditions.

\begin{tabular}{ccc}
\hline Temperature $\left({ }^{\circ} \mathrm{C}\right)$ & ACOF & WSD $(\mathrm{mm})$ \\
\hline 25 & 0.098 & 0.420 \\
50 & 0.081 & 0.430 \\
75 & 0.084 & 0.310 \\
100 & 0.082 & 0.290 \\
\hline \hline Rotating speed (rpm) & ACOF & WSD (mm) \\
\hline 200 & 0.108 & 0.360 \\
800 & 0.100 & 0.365 \\
1,200 & 0.084 & 0.310 \\
\hline
\end{tabular}

that, although changes in temperature had an effect on the tribological properties, anthracite sheets could still effectively reduce friction and the wear extent. The rotating speed had an obvious influence on the tribological performance, as higher rotational speeds resulted in better performance. This may be because the higher rotating speed may increase the rate at which the deposition film on the friction pair renews and reduce the contact time of the friction pair.

\subsection{Surface analysis and lubrication mechanism}

To observe the wear extent, we further characterized the wear tracks of the upper steel ball by SEM, as shown in Fig. 8. The SEM images reveal that a wide wear track with a width of over $576 \mu \mathrm{m}$ formed under the lubrication of only the PAO40 base oil, and the worn steel surface was very rough, exhibiting obvious deep furrows and grooves at high magnification that were indicative of a poor protecting effect (Figs. 8(a) and $8(\mathrm{~b})$ ). There was an improvement in the wear extent of the worn steel surfaces lubricated by oils containing the different additive, especially the oil containing span80-coated anthracite sheets. It was observed that the width of the wear track reduced considerably, the worn surface became smoother, and the furrows and grooves became thinner and shallower, which is indicative of the excellent anti-wear ability of the anthracite sheets.

The worn surfaces of the lower steel ball lubricated

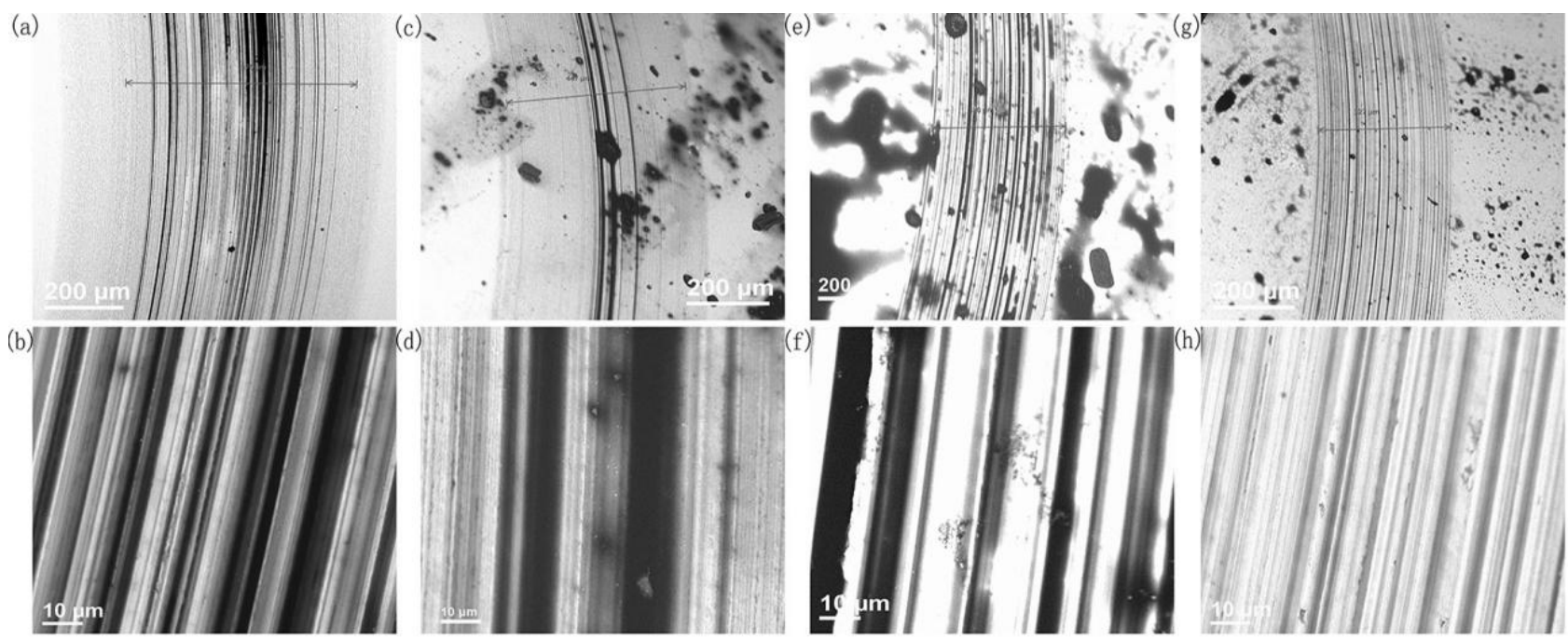

Fig. 8 SEM images of the wear track of of upper steel ball lubricated with: base oil (a, b); oil containing anthracite (c, d); oil containing span80-coated graphite (e, f); oil containing span80-coated anthracite sheets (g, h). 
by different oils were analyzed by FE-SEM. Figure 9(a) shows the morphology of the wear scar when the PAO40 base oil was used. There were obvious wear traces on the worn surface. A magnified view of the highlighted portion in Fig. 9(a) is shown in Fig. 9(b). Tiny wear debris were found to be deposited on the worn surface. However, it can be seen that the worn surface was much smoother and that the wear traces were few; furthermore, a considerable amount of deposition was observed on the worn surface. To acquire information about these depositions, the worn surfaces were analyzed by EDS. As shown in Figs. 10(a) and 10(c), the EDS analysis revealed that the content of $C$ on the worn surface was $18.01 \mathrm{wt} \%$, which may be due to be basal component of the steel ball and carbonization of the base oil. For the oil containing span80-coated anthracite sheet, the content of $C$ on the worn surface was as much as $40.78 \mathrm{wt} \%$. Figure 10(e) shows the elemental mapping of the worn surface in a small area lubricated by oil containing span80-coated anthracite sheets. It was clearly evident that the region of the deposition was deficient in iron and rich in carbon, which confirms that the deposition was due to the anthracite sheets. The results of the FE-SEM and EDS analysis of the worn surface revealed that the span80-coated anthracite sheets could form a regional protective film on the steel surfaces.

The existence of the anthracite sheets on the worn steel surface was confirmed by Raman spectroscopy. Figures 11(a)-11(c) show the OM images of the wear scars on the steel ball, while Fig. 11(d) shows the Raman spectra of the anthracite, PAO40 base oil, base oil lubricated surface, span80-coated graphite lubricated surface, and span80-coated anthracite sheets lubricated surface. The spectrum of the anthracite sheets exhibited characteristic bands: a D-band at $\sim 1,350 \mathrm{~cm}^{-1}$ and a G-band at $1,580 \mathrm{~cm}^{-1}$. The PAO40 base oil is a synthetic oil formed by the polymerization of $\alpha$-dodecene. The Raman spectrum of the base oil contained its characteristic bands: a band at $\sim 1,302 \mathrm{~cm}^{-1}$ and $1,445 \mathrm{~cm}^{-1}$. These distinct bands were detected after the tribotests. For the span80-coated anthracite sheets lubricated surface, the characteristic bands of anthracite, a D-band and G-band, could also be detected, which supported the finding that span80-coated anthracite sheets had been deposited on worn surface to form a deposition film during the friction process..

The Raman spectra reveal that anthracite contributed to the AC performance of the base oil. When only the base oil worked, the Raman spectrum showed the obvious characteristic peaks of iron oxides at $\sim 700 \mathrm{~cm}^{-1}$ [41]. However, the Raman signatures of the span80-coated anthracite lubricated surface did not contain peaks characteristic of iron oxides, indicating that anthracite sheets were able to prevent oxidation on the steel surface.
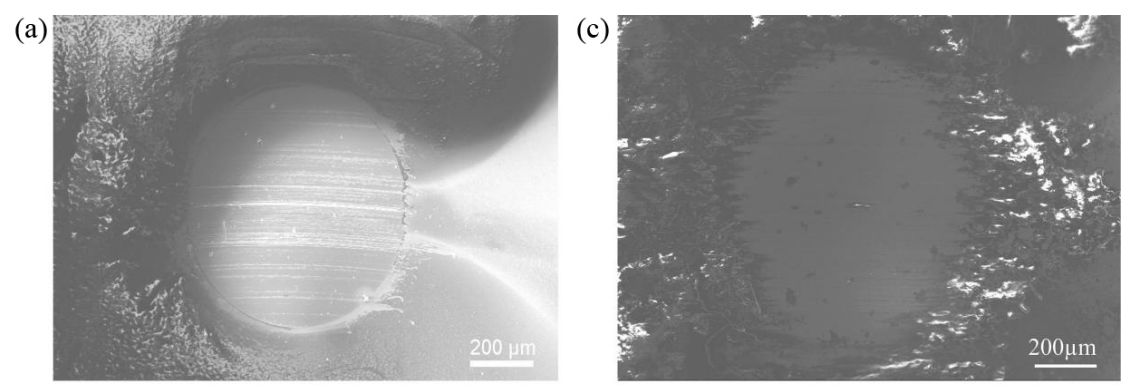

(b)
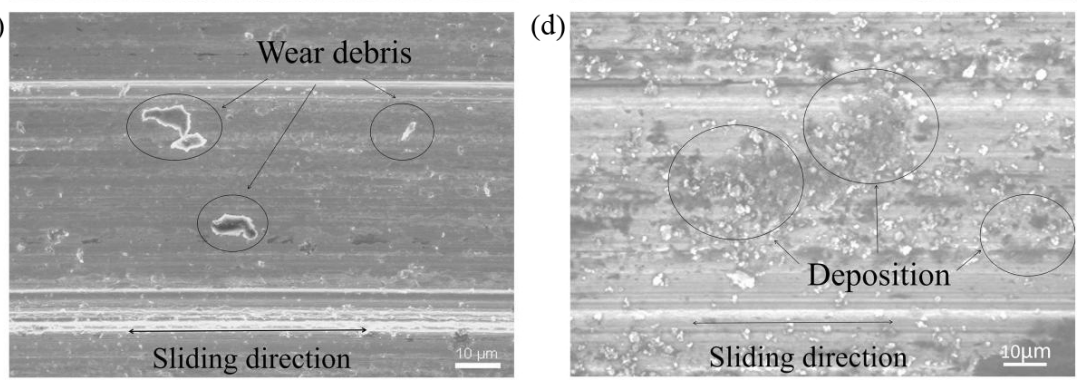

Fig. 9 FE-SEM images for the worn surface lubricated with: base oil (a) and a high magnification (b); oil containing span80-coated anthracite sheets (c) and a high magnification (d). 
(a)

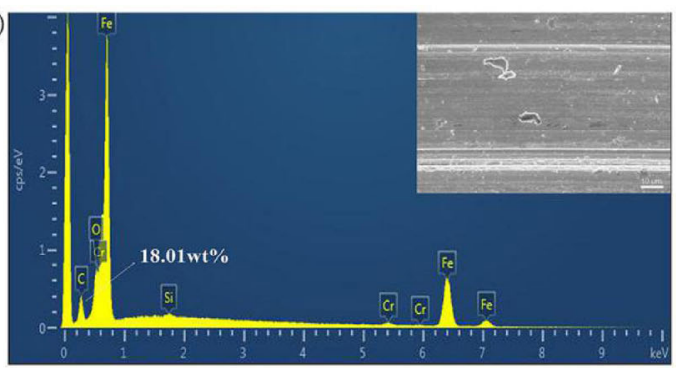

(c) Element Weight (\%) Weight (\%) Atom (\%)

\begin{tabular}{|c|c|c|c|}
\hline Element & wergnt (\%) & $\begin{array}{l}\text { Sigma } \\
\text { Sint }\end{array}$ & Atom (\%) \\
\hline $\mathrm{C}$ & 4.80 & 0.19 & 18.01 \\
\hline $\mathrm{Fe}$ & 88.24 & 1.31 & 71.18 \\
\hline $\mathrm{O}$ & 2.37 & 0.12 & 6.67 \\
\hline $\mathrm{Cr}$ & 4.37 & 1.41 & 3.78 \\
\hline $\mathrm{Si}$ & 0.22 & 0.05 & 0.35 \\
\hline
\end{tabular}

(e)

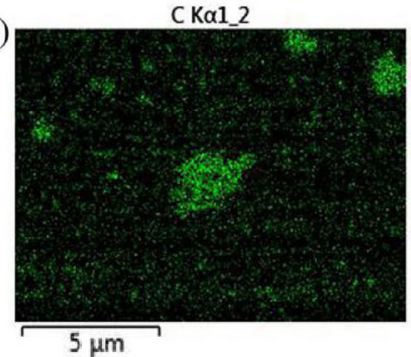

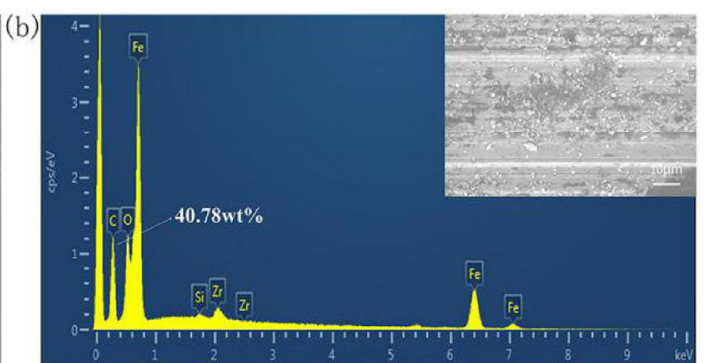

(d) Element Weight (\%) Weight (\%) Atom (\%)

\begin{tabular}{cccc} 
& \multicolumn{3}{c}{ Sigma } \\
\hline $\mathrm{C}$ & 14.69 & 0.28 & 40.78 \\
$\mathrm{Fe}$ & 76.65 & 0.37 & 45.78 \\
$\mathrm{O}$ & 5.86 & 0.14 & 12.22 \\
$\mathrm{Zr}$ & 2.57 & 0.23 & 0.94 \\
$\mathrm{Si}$ & 0.24 & 0.06 & 0.29 \\
\hline
\end{tabular}

$\mathrm{O} K \alpha 1$

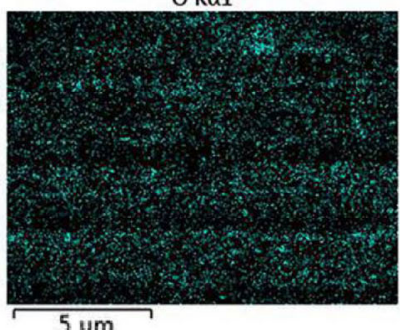

$5 \mu \mathrm{m}$

Fig. 10 EDS pattern of the worn surface lubricated with: base oil (a, c); oil containing span80-coated anthracite sheets (b, d); elemental mapping of deposits on the worn surface (e).
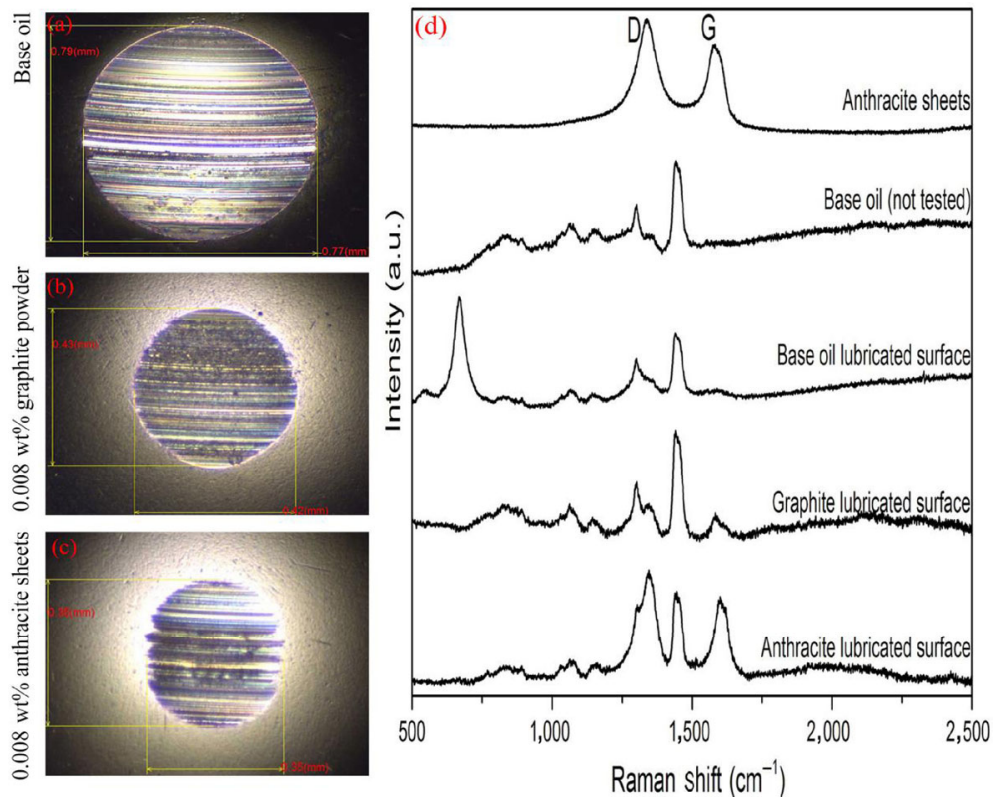

Fig. 11 The OM images of the wear scars on the ball (a-c); Raman spectra for the anthracite sheets, PAO40 base oil, base oil lubricated surface, span80-coated graphite lubricated surface, and the span80-coated anthracite sheets lubricated surface (d).

Figure 12 shows the lubrication mechanism of anthracite sheets schematically. When only the base oil was used, the wear extent of the friction pair was aggravated with the prolongation of the friction time. When anthracite sheets were added, they absorbed onto the surface of the steel ball and formed a protective 


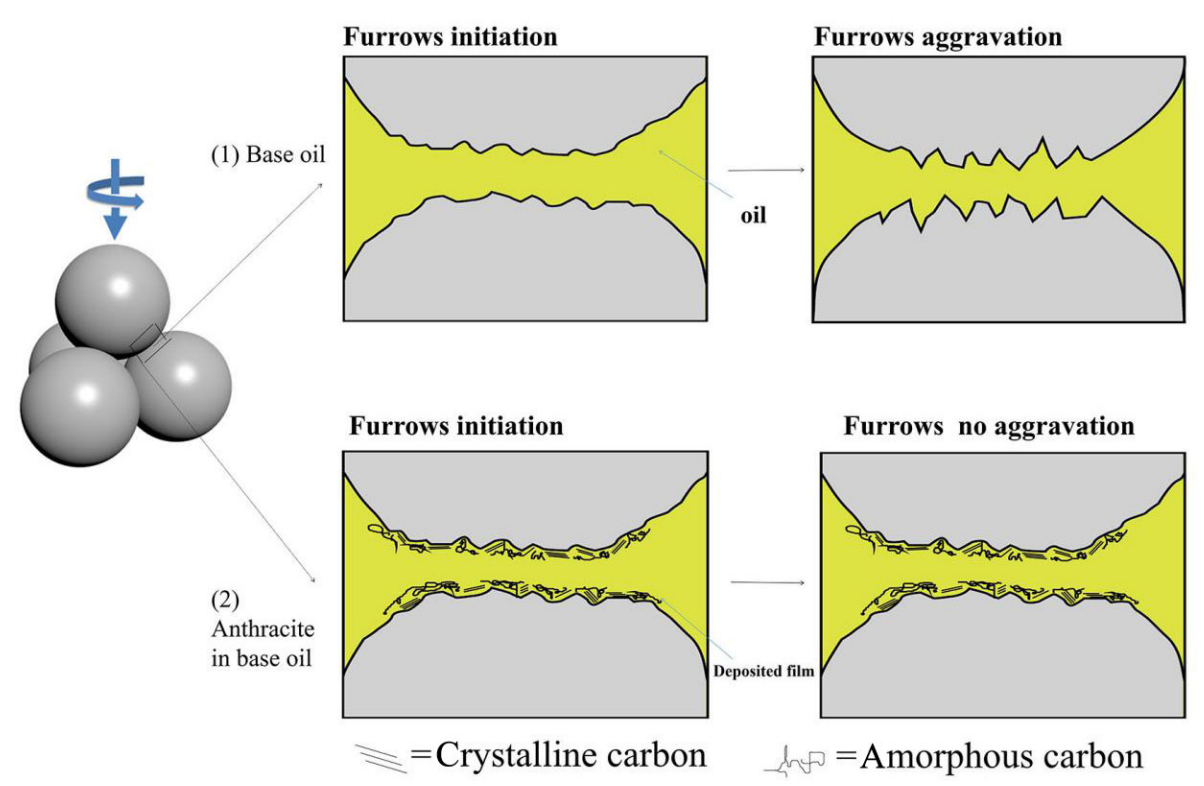

Fig. 12 Schematic diagram of the lubrication mechanism of anthracite sheets as oil additives.

and lubricious film. When subjected to external force, the layers of the crystalline carbon component of anthracite are prone to slip, playing a role in lubrication. At the same time, crystalline carbon has a high strength, which can greatly bear the applied load, thus reducing the wear extent of the friction pair. Although amorphous carbon components do not have regular structures and shapes, their hardness is so large that they can constantly fill and repair the worn parts in the friction process. Therefore, the synergistic action of the two components greatly reduces the wear extent of the friction pair [42].

\section{Conclusions}

Span80-coated anthracite sheets fabricated by a simple ball milling method effectively enhanced the dispersion stability of anthracite in the base oil. Friction and wear tests results demonstrated that span80-coated anthracite sheets possess excellent anti-wear and friction-reducing abilities. The ACOF, WSD, and WV of the oil containing $0.03 \mathrm{wt} \%$ span 80 -coated anthracite sheets were $45.39 \%, 60.13 \%$, and $95.95 \%$ lower, respectively, than those of the base oil at $150 \mathrm{~N}$ and $75{ }^{\circ} \mathrm{C}$. The span80-coated anthracite sheets also exhibited excellent abilities over a wide range of applied loads, temperatures, or rotating speeds. FE-SEM-EDS and Raman spectra analyses confirmed that the lubrication mechanism is attributed to the synergistic action of crystalline and amorphous carbon in anthracite, which protect and mend the surface of the friction pair.

Anthracite sheets not only exhibit excellent tribological performances, but also have the advantages of convenient sources and low cost, which suggest that anthracite has promising potential as a lubricant additive.

\section{Acknowledgements}

This work was financially supported by National Natural Science Foundation of China (No. 51373059), Science and Technology Projects in Fujian Province (Nos. 2017H2001, 2018H0019, and 2018H6012), Science and Technology Innovation Team of Huaqiao University (No. Z14X0046), Subsidized Project for Postgraduates' Innovative Fund in Scientific Research of Huaqiao University, the Graphene Powder \& Composite Research Center, Development and Reform Commission of Fujian Province.

Open Access: This article is licensed under a Creative Commons Attribution 4.0 International License, which permits use, sharing, adaptation, distribution and reproduction in any medium or format, as long as you give appropriate credit to the original author(s) and the source, provide a link to the Creative Commons 
licence, and indicate if changes were made.

The images or other third party material in this article are included in the article's Creative Commons licence, unless indicated otherwise in a credit line to the material. If material is not included in the article's Creative Commons licence and your intended use is not permitted by statutory regulation or exceeds the permitted use, you will need to obtain permission directly from the copyright holder.

To view a copy of this licence, visit http://creativecommons.org/licenses/by/4.0/.

\section{References}

[1] http://www.wtc2017.org/newsinfo/14785, Accessed 15 June 2018.

[2] Wang J, Guo X, He Y, Jiang M, Gu K. Tribological characteristics of graphene as grease additive under different contact forms. Tribol Int 127: 457-469 (2018)

[3] Lee G, Park J, Lee M, Rhee C. Stable dispersion of nanodiamonds in oil and their tribological properties as lubricant additives. Appl Surf Sci 415: 24-27 (2017)

[4] Xie H, Jiang B, He J, Xia X, Pan F. Lubrication performance of $\mathrm{MoS}_{2}$ and $\mathrm{SiO}_{2}$ nanoparticles as lubricant additives in magnesium alloy-steel contacts. Tribol Int 93: 63-70 (2015)

[5] Luo T, Wei X, Huang X. Tribological properties of $\mathrm{Al}_{2} \mathrm{O}_{3}$ nanoparticles as lubricating oil additives. Ceram Int 40: 7143-7149 (2014)

[6] Zhang C, Zhang S M, Song S Y, Yang G B, Yu L G, Wu Z S, Li X H, Zhang P Y. Preparation and tribological properties of surface-capped copper nanoparticales as a water-based lubricant additves. Tribol Lett 54(1): 25-33 (2014)

[7] Wu Y Y, Tsui W C, Liu T C. Experimental analysis of tribological properties of lubricating oils with nanoparticle additives. Wear 262: 819-825 (2007)

[8] Jiang Z Q, Zhang Y J, Yang G B, Yang K P, Zhang S M, Yu L G, Zhang P Y. Triboligical properties of oleylaminemodified ultrabin $\mathrm{WS}_{2}$ nanosheets as the additive in polyalpha olefin over a wide temperature range. Tribol Lett 61(3): 24 (2016)

[9] Rabaso P, Ville F, Dassenoy F, Diaby M, Mogne T L. Boundary lubrication: Influence of the size and structure of inorganic fullerene-like $\mathrm{MoS}_{2}$ nanoparticles on friction and wear reduction. Wear 320(s1-2): 161-178 (2014)

[10] Reeves C J, Menezes P L, Lovell M R, Jen T. The size effect of boron nitride particles on the tribological performance of biolubricants for energy conservation and sustainability. Tribol Lett 51(3): 437-452 (2013)
[11] Huang G W, Yu Q L, Ma Z F, Cai M R, Zhou F, Liu W J. Oil-soluble ionic liquids as antiwear and extreme pressure additives in poly- $\alpha$-olefin for steel/steel contacts. Friction 7(1): 18-31 (2019)

[12] Gonzalez R, Ramos D, Blanco D, Fernandez-Gonzalez A, Viesca J L, Hadfield M, Hernandez-Battez A. Tribological performance of tributylmethylammonium bis (trifluoromethylsulfonyl) amide as neat lubricant and as an additive in a polar oil. Friction 7(3): 282-288 (2019)

[13] Gao X L, Liu D H, Song Z, Dai K. Isosteric design of friction-reduction and anti-wear lubricant additives with less sulfur content. Friction 6(2): 164-182 (2018)

[14] Kumar-Dubey M, Bijwe J, Ramakumar S. PTFE based nano-lubricants. Wear 306(1-2): 80-88 (2013)

[15] Yao Y, Wang X, Guo J, Yang X, Xu B. Tribological property of onion-like fullerenes as lubricant additive. Mater Lett $\mathbf{6 2}$ : 2524-2527 (2008)

[16] Cornelio J, Cuervo P, Hoyos-Palacio L, Lara-Romero J, Toro A. Tribological properties of carbon nanotubes as lubricant additive in oil and water for a wheel-rail system. J Mater Res Technol 5(1): 68-76 (2016)

[17] Peng Y, Hu Y, Wang H. Tribological behaviors of surfactantfunctionalized carbon nanotubes as lubricant additive in water. Tribol Lett 25(3): 247-253 (2007)

[18] Zhang X. Study on taixi anthracite coal to prepare Li-ION battery anode materials. Coal Conversion 38: 75-78 (2015)

[19] Qiu J, Wang Z, Zhao Z. Synthesis of double-walled carbon nanotubes from coal in hydrogen-free atmosphere. Fuel 86(1-2): 282-286 (2017)

[20] Sasikala S P, Henry L, Yesilbag T G. High yield synthesis of aspect ratio controlled graphenic materials from anthracite coal in supercritical fluids. ACS Nano 10(5): 5293-5303 (2016)

[21] Lu J, Li F, Chang P, Xie K. The study of coal based polymer: polyhumic-acid-hexadiamide/ PA6 blend. In 9th International Conference on Coal Science, Pittsburgh, USA, 1997.

[22] Nathalia R M, Cassiano R O, Raissa S R, Rodrigo L L. Azo dye adsorption on anthracite: A view of thermodynamics, kinetics and cosmotropic effects. Sep Purif Technol 209(2-3): 806-814 (2019)

[23] Takagi H, Maruyama K, Yoshizawa N, Yamada Y, Sato Y. $\mathrm{XRD}$ analysis of carbon stacking structure in coal during heat treatment. Fuel 83(3): 2427-2433 (2004)

[24] Lu L, Sahajwalla V, Kong C, Harris D. Quantitative X-ray diffraction analysis and its application to various coals. Carbon 39(12): 1821-1833 (2001)

[25] Lu k. Theoretical analysis of colloidal interaction energy in nanoparticle suspensions. Ceram Int 34(6): 1353-1360 (2008) 
[26] Zhang W, Zhu H, Di Z, Wu D. Research on preparation and friction properties of graphene by liquid phase exfoliation. Nanosci Nanotech 8: 5 (2011)

[27] Smith R J, Lotya M, Coleman J N. The importance of repulsive potential barriers for the dispersion of graphene using surfactants. New J Phys 12(12): 125008 (2010)

[28] Hu E, Hu X, Liu T, Liu Y, Song R, Chen Y. Investigation of morphology, structure and composition of biomass-oil soot particles. Appl Surf Sci 270: 596-603 (2013).

[29] Pei S, Cheng H. The reduction of graphene oxide. Carbon 50(9): 3210-3228 (2012)

[30] Huang D, Chuan X, Cao X. Spectroscopy characterization of anthracite oxide. Spectrosc Spect Anal 36(11): 3698-3703 (2016)

[31] Zhao Y, Zhang Y, Zhang H, Wang Q, Guo Y. Structural characterization of carbonized briquette obtained from anthracite powder. J Anal Appl Pyrolysis 112: 290-297

[32] Lee C, Hwang Y, Park M, Lee J, Choi C, Jung M: Tribological behavior of copper nanoparticles as additives in oil. Curr Appl Phys 9(2): 124-127 (2009)

[33] Bartz W J. Some Investigations on the influence of particle size on the lubricating effectiveness of molybdenum disulfide. ASLE Trans 15: 207-215 (1972)

[34] Shen J, Li N, Shi M, Hu Y, Ye M. Covalent synthesis of organophilic chemically functionalized graphene sheets. J Colloid Interface Sci 348(2): 377-379 (2010)

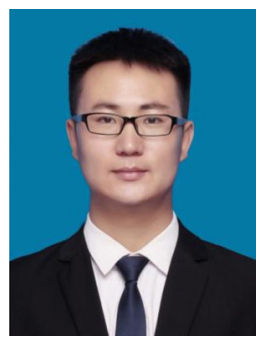

Hailong LIU. He received his bachelor degree in 2017 from Huaqiao University, Xiamen, China. After then, he was a master student in the Graphene Powder and
[35] Ou J, Wang Y, Wang J, Liu S, Li Z P, Yang S R. SelfAssembly of octadecyl trichlorosilane on graphene oxide and the tribological performances of the resultant film. $J$ Phys Chem C 115(20): 10080-10086 (2011)

[36] Dienwiebel M, Verhoeven G S, Pradeep N, Frenken J W, Heimberg J A, Zandbergen H W. Superlubricity of graphite. Phys Rev Lett 92(12): 126101 (2004)

[37] Samyn P, Schoukens G. The lubricity of graphite flake inclusions in sintered polyimides affected by chemical reactions at high temperatures. Carbon 46(7): 1072-1084 (2008)

[38] Berman D, Erdemir A, Sumant A V. Reduced wear and friction enabled by graphene layers on sliding steel surfaces in dry nitrogen. Carbon 59: 167-175 (2013)

[39] Luo J B, Shen M H, Shi B, Wen S Z. Thin film lubrication and lubrication map. Chin J Mech Eng 36(7): 5-10 (2000) (in Chinese).

[40] Zhang W, Zhou M, Zhu H, Tian Y, Wang K L, Wei J Q, Li F, Li Z, Zhang P, Wu D H. Tribological properties of oleic acid-modified graphene as lubricant oil additives. $J$ Phys D: Appl Phys 44: 205303 (2011)

[41] Liang S, Shen Z, Yi M, Liu L, Zhang X Z, Ma S L. In-situ exfoliated graphene for high-performance water-based lubricants. Carbon 96: 1181-1190 (2016)

[42] Wang J, Guo X, He Y, Jiang M, Gu K. Tribological characteristics of graphene as grease additive under different contact forms. Tribol Int 127: 457-469 (2018)

Composite Engineering Center, Huaqiao University. He has recently obtained his master degree in chemical engineering. His research interests include nanomaterials and inorganic mineralogy.

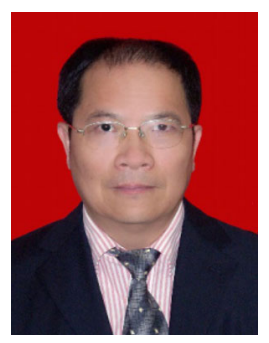

Guohua CHEN. He received his bachelor, M.S., and Ph.D. degrees from Xiamen University, Jilin University, and Tianjin University, China, in 1984, 1987, and 1999,

respectively. He joined in Huaqiao University in 1987. At present, he is a professor in College of Materials Science \& Engineering, Huaqiao University. His research interests are mainly in the graphene preparation and application. 